\title{
Skeletal manifestations of acute lymphoblastic leukemia in two pediatric cases
}

\begin{abstract}
Introduction: Acute leukemia in the pediatric population can present with a variety of orthopedic symptoms and signs, which can lead to delay in diagnosis. We report two such cases that presented to the emergency department in our hospital- King Fahad Medical City-, Riyadh, Saudi Arabia.
\end{abstract}

Objective: The aim of this paper is to put emphasis on the fact that skeletal manifestations may be the first clinical presentation in children suffering from Acute Lymphoblastic Leukemia.

Method: The report describes two pediatric patients who presented to our emergency department primarily with musculoskeletal symptoms but later turned out to be suffering from acute lymphocytic leukemia.

Conclusion: Acute lymphocytic leukemia in pediatric patients can present first to the orthopedic service with bone pain, and nonspecific radiographic features, including: osteopenia, localized bony irregularities and fractures. High index of suspicion is warranted to avoid the delays in the diagnosis of the disease.
Volume 7 Issue I - 2017

\author{
Basma Alfaris,' Osama Alshaya, ${ }^{2}$ Nabil \\ Alassaf, ${ }^{3}$ Bar Hetaimish ${ }^{3}$ \\ 'Medical Intern, College of Medicine, King Saud University, Saudi \\ Arabia \\ ${ }^{2}$ Department of Surgical Specialties, King Fahad Medical City, \\ Saudi Arabia \\ ${ }^{3}$ Department of Orthopedic Surgery, Medical College, Taibah \\ University, Saudi Arabia
} Correspondence: Basma Alfaris, Medical Intern, College of
Medicine, King Saud University, Riyadh, Saudi Arabia, Email basmaalfaris@gmail.com

Received: December 29, 2016 | Published: January 05, 2017

Keywords: Children, Leukemia, Musculoskeletal

\section{Introduction}

Pediatric leukemia is one of the most common pediatric malignancies, representing one quarter of all pediatric cancers. In the USA, the annual incidence is between 3.7 and 4.9 per 100,000 children aged 1-14 years, with peak incidence aged 2-5 years. ${ }^{1}$ Both acute and chronic leukemia can present with a variety of symptoms and signs involving multiple systems in the body. Skeletal involvement in leukaemia was reported during early twentieth century. ${ }^{2}$ These symptoms can involve bones, joints, and soft tissues. For example, bone pain, limping, arthritis, osteomyelitis, transient synovitis, and fractures may all present. This can be considered a diagnostic difficulty when both clinical presentations and abnormal radiographic findings are not traditional characteristics of leukemia patients. ${ }^{3}$ Acute Lymphoblastic Leukemia (ALL) has a high rate of survival $(92+/-7 \%)$ if detected early. ${ }^{4}$

\section{Case I}

A 9-year-old Saudi boy presented to the emergency department complaining of pain in his ankles, with a history of trauma and difficulty walking. He had this trauma one month before reporting to the hospital and was described as trivial by parents. The patient had no history of fever and no change in his weight. On examination, he was afebrile. Neurological exam was normal but he was unable to walk. The X-ray radiograph showed that the posteromedial part of the distal metaphysis of the left tibia had an abnormal configuration with an irregular margin. Furthermore, as shown in Figures 1 \& 2, there was a decrease in tibio-talar joint space bilaterally and a general decrease in bone density. Investigations showed the following values: platelet count: 91; an absolute neutrophil count: 0.2; hemoglobin: 9; and WBC: 2000. Vitamin D, calcium, and bone marrow aspiration tests revealed a marked decrease in the vitamin D level and a $97 \%$ blast in the bone marrow, as shown in Figure 3. Flow cytometry showed positivity for CD19, CD22, CD33, CD34, CD10, CD79a, TdT, CD11b, and HLA-DR. The patient was put on chemotherapy, Tazocin, cotnmoxazol, nystatin, and sodium bicarbonate. Thereafter, his lower limb pain significantly improved and he started to walk normally.

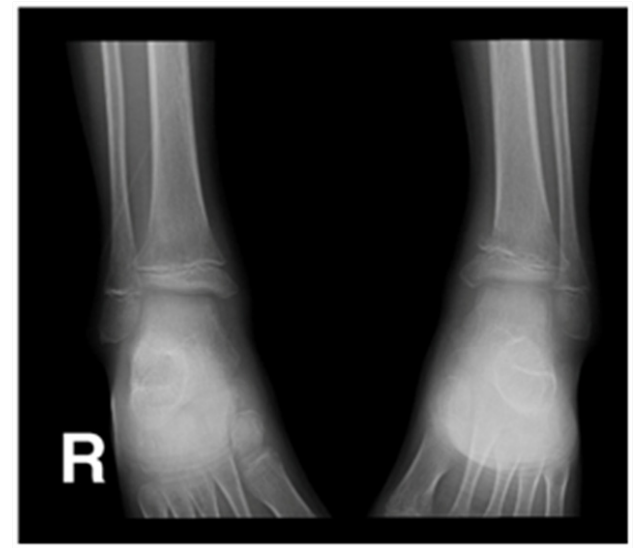

Figure I Anteroposterior view.

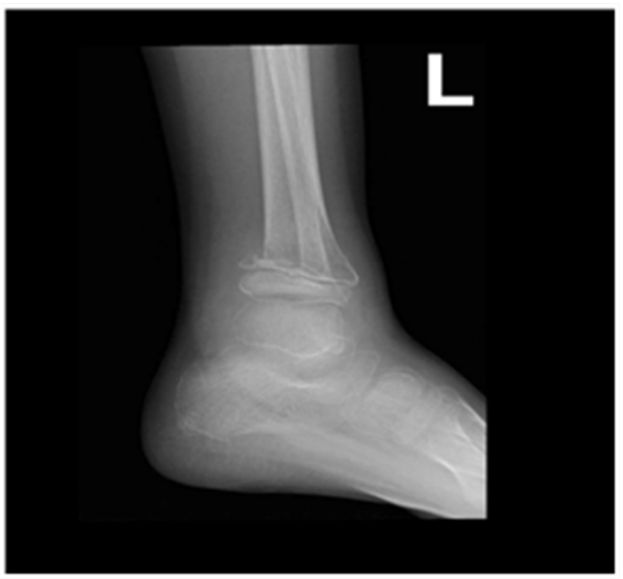

Figure 2 Lateral view

Figures I \& 2 Posteromedial part of the distal metaphysis of the left tibia with abnormal configuration and irregular margin. 


\section{Case 2}

A 12-year-old Saudi girl with a known case of vitamin D deficiency and acute $\beta$-cell lymphoblastic leukemia presented to the emergency department complaining of fever, generalized bone pain for two months, and sternal prominence for one month, with no history of weight loss, masses, or swellings. On examination, she looked pale with generalized bone ache. There was a prominent sternal projection, which was not tender. No obvious lymphadenopathy or hepatosplenomegaly was detected. Further testing showed the following values: CBC: 9.59; absolute neutrophil count: 6.42; hemoglobin: 7.9; platelets: 295; Uric acid: $503 \mu \mathrm{mol} / \mathrm{L}$; LDH: $754 \mu \mathrm{mol} / \mathrm{L}$; Creatinine: $57 \mathrm{mmol} / \mathrm{L}$; calcium: $2.5 \mathrm{mmol} / \mathrm{L}$; alkaline phosphatase: $161 \mathrm{units} / \mathrm{L}$; phosphate: $1.31 \mathrm{mmol} / \mathrm{L}$; and potassium: $3.8 \mathrm{mmol} / \mathrm{L}$. Her T-cell marker and myeloid markers were negative. A CT scan of the chest and abdomen displayed compression fractures extending from $\mathrm{T} 1$ to T6, with diffused mixed lytic and sclerotic osseous changes all over the skeleton, as shown in Figure 4. Moreover, there were multiple low attenuation lesions in the mildly enlarged kidneys. An MRI of the spine showed diffused abnormal heterogeneous appearance of the bone marrow over the entire spine and the sacrum (Figure 5), but no clear cord compression was seen. Bone scintigraphy with $99 \mathrm{mTc}-$ methylene diphosphonate showed diffused increase in tracer uptake in the skeleton. Patchy, geographic like elevated uptake was seen in the skull (Figure 6). The patient was on Tazocin, amikacin and voriconazole, and vitamin D 50,000 IU weekly.

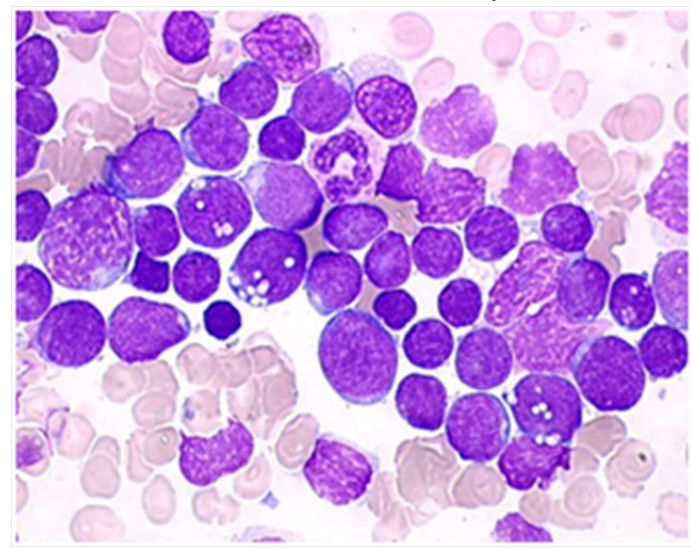

Figure 3 Bone marrow biopsy of acute lymphoblastic leukemia .

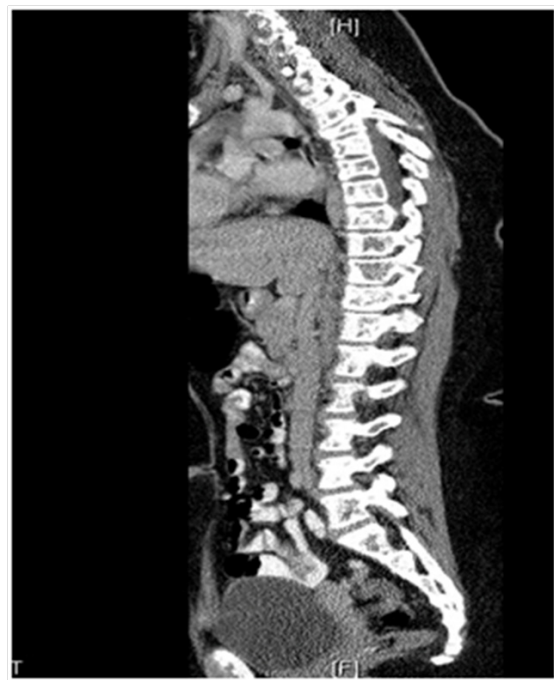

Figure $4 \mathrm{~A} \mathrm{CT}$ scan with compression fractures extending from TI to T6, with diffused mixed lytic and sclerotic osseous changes all over the skeleton.

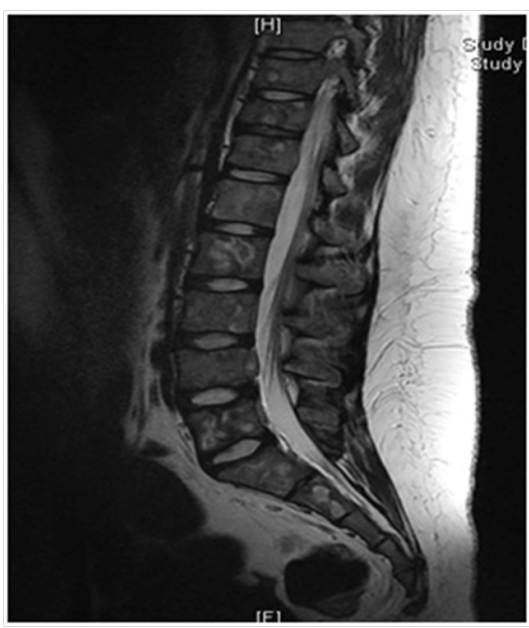

Figure 5 An MRI showing diffused abnormal heterogeneous appearance of the bone marrow over the entire spine and the sacrum.

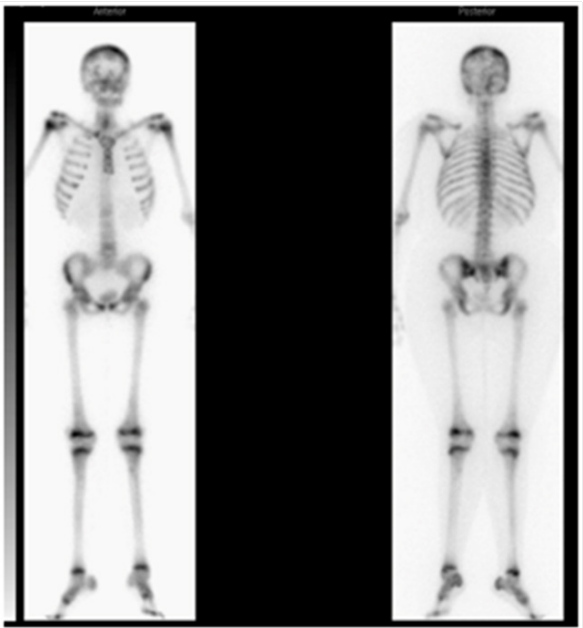

Figure 6 Bone scintigraphy with 99mTc-methylene diphosphonate showing diffuse increase in tracer uptake in the skeleton and patchy, geographic like elevated uptake in the skull.

\section{Discussion}

ALL has a variety of clinical and radiological orthopedic manifestations in the pediatric population. Riccio et al. retrospectively reviewed 328 patients of acute leukemia. 22\% presented with musculoskeletal manifestations. ${ }^{5}$ Reported radiographic features in descending order of frequency includes: osteoporosis, fractures, osteolysis, osteosclerosis, periosteal reactions and metaphyseal bands. Sinigaglia reviewed 122 cases of acute leukemia (AL) in children to evaluate the incidence of different orthopedic features. ${ }^{6} \mathrm{At}$ presentation, 102 cases diagnosed with ALL and 20 cases diagnosed with acute myeloid leukemia. Of the cases studied, $38.3 \%$ presented with musculoskeletal symptoms. Clinically, $34.4 \%$ presented with pain, $22.9 \%$ with impairment of the function, $12.3 \%$ with limping, $10.6 \%$ with swelling, and $5.7 \%$ showed joint effusion.

Of the musculoskeletal cases, $40.2 \%$ had at least one abnormal radiological feature, $13.1 \%$ had osteolysis, $9.8 \%$ had metaphyseal bands, 9\% had osteopenia, 7.4\% had osteosclerosis, $5.7 \%$ had a permeative pattern, $5.7 \%$ had pathological fractures, $4.1 \%$ had periosteal reactions, and $2.5 \%$ had mixed lysis-sclerosis lesions.

Our case report is the first to discuss the unusual sign of abnormal bony configurations with irregular margin in ALL patients. This should 
lead to further investigation in a larger ALL pediatric population. We believe that this sign might facilitate early diagnosis of these cases. Consequently, early diagnosis can significantly decrease the morbidity and mortality of ALL. ${ }^{4,6}$ However, the generalized decrease in bone density is commonly seen in ALL patients. Cohan et al. reported a case of ALL with multiple bone fractures associated with a decrease in bone mineral density (BMD). ${ }^{7}$ They identified the relationship between ALL and decreased BMD as multifactorial. This involves three factors: (1) the dissemination of malignant cells' soluble products ${ }^{8}{ }^{8}$ (2) leukemia cells invading the bones, and (3) the countereffect of the treatment used for ALL (corticosteroid, methotrexate, and radiation)..$^{9,10}$

Our first case is aligned with the first two factors as a cause behind the decrease in BMD, since our case at presentation did not receive any of the treatment described in factor 3 .

In case 2 , the images showed compression fractures extending from T1 to T6, with diffused mixed lytic and sclerotic osseous changes all over the skeleton. Moreover, an MRI showed the diffused abnormal heterogeneous appearance of the bone marrow over the entire spine and the sacrum. These findings conform to what is documented in the literature and the orthopedic doctors who find such signs should suspect leukemia, which can still be diagnosed at an early stage for a good prognosis and a chance to save lives.

\section{Conclusion}

ALL is one of the most common pediatric malignancies, and it has a high rate of survival $(92+/-7 \%)$ if detected early. Therefore, orthopedic surgeons have a major role to play in the management and survival of ALL patients through early diagnosis of their musculoskeletal manifestations.

\section{Conflicts of interest}

None.

\section{References}

1. Ribera JM, Oriol A. Acute lymphoblastic leukemia in adolescents and young adults. Hematol Oncol Clin North Am. 2009;23(5):1033-1042.

2. Snelling C, Brown A. Bone changes in leukaemia: Part I.-Clinical and roentgenological. Arc Dis Child. 1934;9(53):315-326.

3. Muwakkit S, Al-Aridi C, Samra A, et al. Implementation of an intensive risk-stratified treatment protocol for children and adolescents with acute lymphoblastic leukemia in Lebanon. Am J Hematol. 201287(7):678-683.

4. Simone JV. History of the treatment of childhood ALL: a paradigm for cancer cure. Best Pract Res Clin Haematol. 200619(2):353-359.

5. Riccio I, Marcarelli M, Del Regno N, et al. Musculoskeletal problems in pediatric acute leukemia. J Pediatr Orthop B. 201322(3):264-269.

6. Sinigaglia R, Gigante C, Bisinella G, et al. Musculoskeletal manifestations in pediatric acute leukemia. J Pediatr Orthop. 200828(1):20-28.

7. Cohan N, Sarikhani S, Moslemi S, et al. Initial presentation of acute lymphoblastic leukemia with osteoporosis and multiple spontaneous bone fractures. Iran Red Crescent Med J. 2011;13(1):52.

8. Kostić G, Đurić Z, Bunjevački G, et al. Bone changes, mineral homeostasis in childhood acute lymphoblastic leukemia. Facta Universitatis. 200411(3):123-126.

9. Haddy TB, Mosher RB, Reaman GH. Osteoporosis in survivors of acute lymphoblastic leukemia. The oncologist. 20016(3):278-285.

10. Karimi M, Mehrabani D, Yarmohammadi H, et al. The prevalence of signs and symptoms of childhood leukemia and lymphoma in Fars Province, Southern Iran. Cancer Detect Prev. 200832(2):178-183.

\section{Acknowledgments}

None. 